JOURNAL OF

FUNCTION SPACES AND APPLICATIONS

Volume 8, Number 1 (2010), 1-16
(C) 2010, Scientific Horizon http://www.jfsa.net

\title{
Marcinkiewicz integrals with variable kernels on Hardy and weak Hardy spaces*
}

\section{Xiangxing Tao, Xiao Yu and Songyan Zhang}

(Communicated by Maria Carro)

2000 Mathematics Subject Classification. 42B20, 42B30.

Keywords and phrases. Marcinkiewicz integral, variable kernel, Hardy space, weak Hardy space, $L^{1, \alpha}$-Dini condition.

Abstract. In this article, we consider the Marcinkiewicz integrals with variable kernels defined by

$$
\mu_{\Omega}(f)(x)=\left(\int_{0}^{\infty}\left|\int_{|x-y| \leq t} \frac{\Omega(x, x-y)}{|x-y|^{n-1}} f(y) d y\right|^{2} \frac{d t}{t^{3}}\right)^{1 / 2}
$$

where $\Omega(x, z) \in L^{\infty}\left(\mathbb{R}^{n}\right) \times L^{q}\left(\mathbb{S}^{n-1}\right)$ for $q>1$. We prove that the operator $\mu_{\Omega}$ is bounded from Hardy space, $H^{p}\left(\mathbb{R}^{n}\right)$, to $L^{p}\left(\mathbb{R}^{n}\right)$ space; and is bounded from weak Hardy space, $H^{p, \infty}\left(\mathbb{R}^{n}\right)$, to weak $L^{p}\left(\mathbb{R}^{n}\right)$ space for $\max \left\{\frac{2 n}{2 n+1}, \frac{n}{n+\alpha}\right\}<p<1$, if $\Omega$ satisfies the $L^{1, \alpha}$-Dini condition with any $0<\alpha \leq 1$.

\section{Introduction}

Let $\mathbb{R}^{n}(n \geq 2)$ be the $n$-dimensional Euclidean space and $\mathbb{S}^{n-1}$ denote the unit sphere in $\mathbb{R}^{n}$ equipped with induced Lebesgue measure $d \sigma$, and let $x^{\prime}=\frac{x}{|x|}$ for any $x \neq 0$.

* This work was supported partly by National Natural Science Foundation of China under grant \#10771110, NSF of Ningbo City under grant \#2006A610090, and sponsored by SRF for ROCS, SEM. 
In 1958, E. M. Stein [12] first introduced the following Marcinkiewicz integral $\mu_{\omega}$ of higher dimension with convolution kernel,

$$
\mu_{\omega}(f)=\left(\int_{0}^{\infty}\left|\int_{|x-y| \leq t} \frac{\omega(x-y)}{|x-y|^{n-1}} f(y) d y\right|^{2} \frac{d t}{t^{3}}\right)^{1 / 2},
$$

where $\omega(x)$ is a homogeneous function of degree zero with $\omega \in L^{1}\left(\mathbb{S}^{n-1}\right)$ and $\int_{\mathbb{S}^{n-1}} \omega\left(x^{\prime}\right) d \sigma\left(x^{\prime}\right)=0$.

E. M. Stein proved that $\mu_{\omega}$ is bounded on $L^{p}\left(\mathbb{R}^{n}\right)$ for $1<p \leq 2$ and is of weak type $(1,1)$ if $\omega \in \operatorname{Lip}_{\alpha}\left(\mathbb{S}^{n-1}\right)$ with $0<\alpha \leq 1$. Subsequently, A. Benedek, A. Calderon and R. Panzone [3] showed that if $\omega \in C^{1}\left(\mathbb{S}^{n-1}\right)$, then $\mu_{\omega}$ is bounded on $L^{p}\left(\mathbb{R}^{n}\right)$ for $1<p<\infty$. Later on, the above results were improved by many authors under some weaker smoothness conditions on $\omega$, see $[1],[7,8],[11],[14,15,16]$ for instance.

We remark that the Marcinkiewicz integral is essentially a LittlewoodPaley $g$-function. If let $\phi(x)=\omega(x)|x|^{-n+1} \chi_{B}(x)$ and $\phi_{t}(x)=t^{-n} \phi(x / t)$, where $B$ denotes the unit ball of $\mathbb{R}^{n}$ and $\chi_{B}$ denotes the characteristic function of $B$, then

$$
\mu_{\omega}(f)(x)=\left(\int_{0}^{\infty}\left|\phi_{t} * f(x)\right|^{2} \frac{d t}{t}\right)^{1 / 2}=g_{\phi}(f)(x) .
$$

In order to study non-smoothness partial differential equations with variable coefficients, mathematicians pay more attention to the singular integral with variable kernels, see [2], [4], [5] and [6] among others. Specially, in 1955 Calderón and Zygmund [4] considered the singular integral with variable kernel defined by

$$
T_{\Omega}(f)(x)=p . v \cdot \int_{\mathbb{R}^{n}} \frac{\Omega(x, x-y)}{|x-y|^{n}} f(y) d y .
$$

In this paper, we study the Marcinkiewicz integral with variable kernel defined by

$$
\mu_{\Omega}(f)(x)=\left(\int_{0}^{\infty}\left|\int_{|x-y| \leq t} \frac{\Omega(x, x-y)}{|x-y|^{n-1}} f(y) d y\right|^{2} \frac{d t}{t^{3}}\right)^{1 / 2} .
$$

We point out that $\mu_{\Omega}$ can be interpreted as a Hilbert-valued function. In fact, denote the Hilbert space $\mathcal{H}$ by 


$$
\mathcal{H}=\left\{h(t):\|h\|_{\mathcal{H}}=\left(\int_{0}^{\infty}|h(t)|^{2} \frac{d t}{t}\right)^{1 / 2}<+\infty\right\},
$$

and let $h_{f}(t, x)=t^{-1} F_{\Omega, t}(x)$, where

$$
F_{\Omega, t}(x)=\int_{|x-y| \leq t} \frac{\Omega(x, x-y)}{|x-y|^{n-1}} f(y) d y .
$$

Then we obtain that $\mu_{\Omega}(f)(x)=\left\|h_{f}(\cdot, x)\right\|_{\mathcal{H}}$.

Before stating our theorems, we first introduce some definitions about the variable kernel $\Omega(x, z)$. A function $\Omega(x, z)$ defined on $\mathbb{R}^{n} \times \mathbb{R}^{n}$ is said to be in $L^{\infty}\left(\mathbb{R}^{n}\right) \times L^{q}\left(\mathbb{S}^{n-1}\right), q \geq 1$, if $\Omega(x, z)$ satisfies the following three conditions:

(1) $\Omega(x, \lambda z)=\Omega(x, z)$, for any $x, z \in \mathbb{R}^{n}$ and any $\lambda>0$;

(2) $\|\Omega\|_{L^{\infty}\left(\mathbb{R}^{n}\right) \times L^{q}\left(\mathbb{S}^{n-1}\right)}=\sup _{r \geq 0, y \in \mathbb{R}^{n}}\left(\int_{\mathbb{S}^{n-1}}\left|\Omega\left(r z^{\prime}+y, z^{\prime}\right)\right|^{q} d \sigma\left(z^{\prime}\right)\right)^{1 / q}<$ $\int_{\mathbb{S}^{n-1}}^{\infty} \Omega\left(x, z^{\prime}\right) d \sigma\left(z^{\prime}\right)=0$ for any $x \in \mathbb{R}^{n}$.

In [4], Calderón and Zygmund proved that if $\Omega$ satisfies (1), (3) and

$$
\sup _{y \in \mathbb{R}^{n}}\left(\int_{\mathbb{S}^{n-1}}\left|\Omega\left(y, z^{\prime}\right)\right|^{q} d \sigma\left(z^{\prime}\right)\right)^{1 / q}<\infty,
$$

then $T_{\Omega}$ is bounded on $L^{2}\left(\mathbb{R}^{n}\right)$ provided $q \geq 2(n-1) / n$. They also found that for no $n$ can we replace the exponent $2(n-1) / n$ by a smaller one. Since the condition (2) implies (2'), so the $L^{2}\left(\mathbb{R}^{n}\right)$ boundedness of $T_{\Omega}$ holds if $\Omega \in L^{\infty}\left(\mathbb{R}^{n}\right) \times L^{q}\left(\mathbb{S}^{n-1}\right)$ with $q \geq 2(n-1) / n$. Recently in [7], the authors proved that if $\Omega \in L^{\infty}\left(\mathbb{R}^{n}\right) \times L^{q}\left(\mathbb{S}^{n-1}\right)$ with $q \geq 2(n-1) / n$, then $\mu_{\Omega}$ is bounded on $L^{2}\left(\mathbb{R}^{n}\right)$; and they also showed the $H^{1}-L^{1}$ boundedness of $\mu_{\Omega}$ under certain Dini condition.

For $0<\alpha \leq 1$, a function $\Omega \in L^{\infty}\left(\mathbb{R}^{n}\right) \times L^{1}\left(\mathbb{S}^{n-1}\right)$ is called to satisfy the $L^{1, \alpha}$-Dini condition if

$$
\int_{0}^{1} \frac{\varpi(\delta)}{\delta^{1+\alpha}} d \delta<\infty
$$

where

$$
\varpi(\delta)=\sup _{r>0, y \in \mathbb{R}^{n}|O|<\delta} \int_{\mathbb{S}^{n-1}}\left|\Omega\left(r z^{\prime}+y, O z^{\prime}\right)-\Omega\left(r z^{\prime}+y, z^{\prime}\right)\right| d \sigma\left(z^{\prime}\right),
$$

and $O$ is a rotation in $\mathbb{R}^{n}$ with $|O|=\|O-I\|$, where $I$ is the identity operator. For the special case $\alpha=0$, it reduces to the $L^{1}$-Dini condition 
Our first aim is to show that the Marcinkiewicz integral $\mu_{\Omega}$ with variable kernel is bounded on Hardy spaces $H^{p}\left(\mathbb{R}^{n}\right)$ with some $p<1$.

Theorem 1.1. Let $\Omega(x, z) \in L^{\infty}\left(\mathbb{R}^{n}\right) \times L^{q}\left(\mathbb{S}^{n-1}\right)$ with $q>2(n-1) / n$, and let $\Omega\left(x, z^{\prime}\right)$ satisfy the $L^{1, \alpha}$-Dini condition with $0<\alpha \leq 1$. Then, if $\max \left\{\frac{2 n}{2 n+1}, \frac{n}{n+\alpha}\right\}<p<1$, there exists an absolute constant $C$ independent of $f$ such that

$$
\left\|\mu_{\Omega}(f)\right\|_{L^{p}\left(\mathbb{R}^{n}\right)} \leq C\|f\|_{H^{p}\left(\mathbb{R}^{n}\right)} .
$$

Corollary 1.2. Let $\omega(z) \in L^{q}\left(\mathbb{S}^{n-1}\right)$ with $q>2(n-1) / n$, and let $\omega\left(z^{\prime}\right)$ satisfy $L^{1, \alpha}$-Dini condition with $0<\alpha \leq 1$. Then, $\mu_{\omega}$ is bounded from Hardy space $H^{p}\left(\mathbb{R}^{n}\right)$ to $L^{p}\left(\mathbb{R}^{n}\right)$ for $\max \left\{\frac{2 n}{2 n+1}, \frac{n}{n+\alpha}\right\}<p<1$.

Another aim of the paper is to derive that $\mu_{\Omega}$ is bounded from weak Hardy space $H^{p, \infty}\left(\mathbb{R}^{n}\right)$ to weak $L^{p}\left(\mathbb{R}^{n}\right)$ space, $L^{p, \infty}\left(\mathbb{R}^{n}\right)$, for some $p<1$. Let us first recall the definition of weak Hardy space $H^{p, \infty}\left(\mathbb{R}^{n}\right)$.

Definition 1.3. Let $\varphi \in C_{0}^{\infty}$ with $\int \varphi(x) d x \neq 0$. Denote by $f_{+}^{*}(x)=$ $\sup _{t>0}\left|\left(\varphi_{t} * f\right)(x)\right|$, where $\varphi_{t}(x)=t^{-n} \varphi\left(\frac{x}{t}\right)$. A distribution $f$ is said to belong to the weak Hardy space $H^{p, \infty}\left(\mathbb{R}^{n}\right)$ if $f_{+}^{*}(x)$ belongs to the weak $L^{p}\left(\mathbb{R}^{n}\right)$ space, $L^{p, \infty}\left(\mathbb{R}^{n}\right)$, i.e, there is a constant $C>0$ such that

$$
\left|\left\{x \in R^{n}: f_{+}^{*}(x)>\beta\right\}\right| \leq \frac{C^{p}}{\beta^{p}}, \quad \forall \beta>0 .
$$

The smallest constant $C$ satisfying the above inequality is called the $H^{p, \infty}$ norm of $f$, and is denoted by $\|f\|_{H^{p, \infty}}$.

Theorem 1.4. Let $\Omega(x, z) \in L^{\infty}\left(\mathbb{R}^{n}\right) \times L^{q}\left(\mathbb{S}^{n-1}\right)$ with $q>2(n-1) / n$, and let $\Omega\left(x, z^{\prime}\right)$ satisfy the $L^{1, \alpha}$-Dini condition with $0<\alpha \leq 1$. Then, if $\max \left\{\frac{2 n}{2 n+1}, \frac{n}{n+\alpha}\right\}<p<1$, there exists a constant $C$ independent of $f$ and $\beta$ such that

$$
\left|\left\{x:\left|\mu_{\Omega} f(x)\right|>\beta\right\}\right| \leq C \frac{\|f\|_{H^{p, \infty}}^{p}}{\beta^{p}} .
$$

Corollary 1.5. Let $\omega(z) \in L^{q}\left(\mathbb{S}^{n-1}\right)$ for $q>2(n-1) / n$, and let $\omega\left(z^{\prime}\right)$ satisfy $L^{1, \alpha}$-Dini condition with $0<\alpha \leq 1$. Then $\mu_{\omega}$ is bounded from weak Hardy space $H^{p, \infty}\left(\mathbb{R}^{n}\right)$ to weak space $L^{p, \infty}\left(\mathbb{R}^{n}\right)$ for $\max \left\{\frac{2 n}{2 n+1}, \frac{n}{n+\alpha}\right\}<p<$ 1 .

Remark 1.6. It's easy to see that $\operatorname{Lip}_{\alpha} \subset L^{1, \alpha}$-Dini for any $0<\alpha \leq 1$. Thus, the conclusions of Corollary 1.2 and 1.5 may be regarded as an improvement and extension of Stein's results about the Marcinkiewicz integrals with convolution kernels in [12] and [8]. 
Remark 1.7. It is worthy noting that the $H^{1}-L^{1}$ boundedness of $\mu_{\Omega}$ may be regarded as the limit case of Theorem 1.1 by choosing $p=1$ and letting $\alpha \rightarrow 0$. Hence, Theorem 1.3 and Corollary 1.7 in [7] are the special cases of above Theorem 1.1.

Throughout the paper, $C$ always denotes a positive constant not necessarily the same at each occurrence. We use $a \sim b$ to mean the equivalence of $a$ and $b$; that is, there exists a positive constant $C$ independent of $a, b$ such that $C^{-1} a \leq b \leq C a$.

\section{Proof of theorem 1.1}

In order to show the $H^{p}-L^{p}$ boundedness of $\mu_{\Omega}$, we will use the atomic decomposition theory of the real Hardy space $H^{p}\left(\mathbb{R}^{n}\right)$ for $\frac{n}{n+1}<p \leq 1$, see for instance [13]. A function $a(x)$ is said to be $(p, 2,0)$ atom if it satisfies the following three conditions:

(i) $\operatorname{supp}(a) \subset B\left(x_{0}, r\right)$, where $B\left(x_{0}, r\right)=\left\{y \in \mathbb{R}^{n}:\left|y-x_{0}\right| \leq r\right\}$ is a ball in $\mathbb{R}^{n}$;

(ii) $\|a\|_{L^{2}} \leq\left|B\left(x_{0}, r\right)\right|^{1 / 2-1 / p}$;

(iii) $\int_{\mathbb{R}^{n}} a(x) d x=0$.

It is well known that every $f \in H^{p}\left(\mathbb{R}^{n}\right), \frac{n}{n+1}<p \leq 1$, has an atomic decomposition $f=\sum_{k} \lambda_{k} a_{k}$, which converges in $H^{p}$ norm and in the sense of distributions; where $\left(\sum_{k}\left|\lambda_{k}\right|^{p}\right)^{1 / p} \cong\|f\|_{H^{p}}$, and all $a_{k}(x)$ are $(p, 2,0)$ atoms.

Start with $f$ in a nice dense class of function, say $f \in H^{p}\left(\mathbb{R}^{n}\right) \cap C_{0}^{\infty}\left(\mathbb{R}^{n}\right)$. If we denote the kernel by $K(x, z)=\frac{\Omega(x, z)}{|z|^{n-1}}$ and set

$$
\mu_{\Omega, \varepsilon}(f)(x)=\left(\int_{0}^{\infty}\left|F_{\Omega, t, \varepsilon}(x)\right|^{2} \frac{d t}{t^{3}}\right)^{\frac{1}{2}}
$$

where

$$
F_{\Omega, t, \varepsilon}(x)=\int_{\varepsilon<|x-y| \leq t} K(x, x-y) f(y) d y .
$$

Now following [13] (Page 115), we write the distribution kernel $K=$ $K_{0}+K_{\infty}$, where $K_{0}$ has compact support, and thus the distribution $F_{\Omega, t, \varepsilon}$ is well defined for every fixed $\varepsilon$ and $t$, and

$$
F_{\Omega, t, \varepsilon}(x)=\sum_{k} \lambda_{k} \int_{\varepsilon<|x-y| \leq t} K(x, x-y) a_{k}(y) d y .
$$


We claim that, for almost every $x \in \mathbb{R}^{n}, \mu_{\Omega}(f)(x)=\lim _{\varepsilon \rightarrow 0} \mu_{\Omega, \varepsilon}(f)(x)$. To see the claim, we use the cancellation condition of $\Omega$ and the fact $\Omega(x, z) \in L^{\infty}\left(\mathbb{R}^{n}\right) \times L^{r}\left(\mathbb{S}^{n-1}\right)$ for any $1 \leq r<2(n-1) / n$ to obtain

$$
\begin{aligned}
F_{\Omega, t, \varepsilon}(x) & =\int_{\varepsilon<|x-y| \leq t} \frac{\Omega(x, x-y)}{|x-y|^{n-1}}(f(y)-f(x)) d y \\
& \leq C \int_{|x-y| \leq t} \frac{|\Omega(x, x-y)|}{|x-y|^{n-2}} d y \leq C t^{2} .
\end{aligned}
$$

On the other hand, Hölder inequality gives

$$
\begin{aligned}
F_{\Omega, t, \varepsilon}(x) & \leq C\left(\int_{|x-y| \leq t} \frac{|\Omega(x, x-y)|^{r}}{|x-y|^{(n-1) r}} d y\right)^{1 / r} \\
& \leq C t^{-(n-1)+(n / r)}
\end{aligned}
$$

for any $1<r<n /(n-1)$. Therefore

$$
t^{-3}\left|F_{\Omega, t, \varepsilon}(x)\right|^{2} \leq C\left(t \chi_{(0,1)}(t)+t^{-1-2 n+(2 n / r)} \chi_{(1, \infty)}(t)\right)
$$

uniformly on $\varepsilon$. So by the Lebesgue dominated convergence theorem we get that $\mu_{\Omega}(f)(x)=\lim _{\varepsilon \rightarrow 0} \mu_{\Omega, \varepsilon}(f)(x)$.

Thus, by similar approximation arguments as in [9] (Theorem 7.3) and in [13] (Page 115), we can obtain

$$
\mu_{\Omega}(f) \leq \sum_{k}\left|\lambda_{k}\right| \mu_{\Omega}\left(a_{k}\right)
$$

Therefore, to derive the inequality (1.3) for any $f \in H^{p}\left(\mathbb{R}^{n}\right)$ and prove Theorem 1.1, it suffices to show that for any $(p, 2,0)$ atom $a(x)$, there exists a constant $C>0$ independent of $a(x)$ such that

$$
\left\|\mu_{\Omega}(a)\right\|_{L^{p}}^{p} \leq C .
$$

Without loss of generality, we let the support of the atom $a(x)$ is $B=B(0, r)$, and denote $B^{*}=B(0,8 r)$. Using the $L^{2}$ boundedness of $\mu_{\Omega}$, we have

$$
\begin{gathered}
\int_{B^{*}}\left|\mu_{\Omega}(a)(x)\right|^{p} d x \leq\left|B^{*}\right|^{1-p / 2}\left\|\mu_{\Omega}(a)\right\|_{L^{2}}^{p} \\
\leq C\left|B^{*}\right|^{1-p / 2}\|a\|_{L^{2}}^{p} \quad \leq C .
\end{gathered}
$$


Note that $(u+v)^{s} \leq u^{s}+v^{s}$ for any $u, v \geq 0$ and $0 \leq s \leq 1$. It is left to give the estimate for the integral

$$
\begin{aligned}
I= & \int_{\left(B^{*}\right)^{c}}\left|\mu_{\Omega}(a)(x)\right|^{p} d x \\
= & \int_{\left(B^{*}\right)^{c}}\left(\int_{0}^{\infty}\left|\int_{|x-y| \leq t} \frac{\Omega(x, x-y)}{|x-y|^{n-1}} a(y) d y\right|^{2} \frac{1}{t^{3}} d t\right)^{p / 2} d x \\
\leq & \int_{\left(B^{*}\right)^{c}}\left(\int_{0}^{|x|+2 r}\left|\int_{|x-y| \leq t} \frac{\Omega(x, x-y)}{|x-y|^{n-1}} a(y) d y\right|^{2} \frac{1}{t^{3}} d t\right)^{p / 2} d x \\
& +\int_{\left(B^{*}\right)^{c}}\left(\int_{|x|+2 r}^{\infty}\left|\int_{|x-y| \leq t} \frac{\Omega(x, x-y)}{|x-y|^{n-1}} a(y) d y\right|^{2} \frac{1}{t^{3}} d t\right)^{p / 2} d x \\
:= & I_{1}+I_{2} .
\end{aligned}
$$

We note that, for $y \in B$ and $x \in\left(B^{*}\right)^{c},|x-y| \sim|x| \sim|x|+2 r$. Thus by the mean value theorem we have

$$
\left|\frac{1}{|x-y|^{2}}-\frac{1}{(|x|+2 r)^{2}}\right| \leq C \frac{r}{|x-y|^{3}}
$$

Applying this inequality and the Minkowski's inequality, we obtain that

$$
\begin{aligned}
& I_{1}=\int_{\left(B^{*}\right)^{c}}\left(\int_{0}^{|x|+2 r}\left|\int_{|x-y| \leq t} \frac{\Omega(x, x-y)}{|x-y|^{n-1}} a(y) d y\right|^{2} \frac{1}{t^{3}} d t\right)^{p / 2} d x \\
& \leq C \int_{\left(B^{*}\right)^{c}}\left(\int_{B}\left(\int_{|x-y|}^{|x|+2 r} \frac{|\Omega(x, x-y)|^{2}}{|x-y|^{2(n-1)}}|a(y)|^{2} \frac{1}{t^{3}} d t\right)^{1 / 2} d y\right)^{p} d x \\
& =C \int_{\left(B^{*}\right)^{c}}\left(\int_{B} \frac{|\Omega(x, x-y)|}{|x-y|^{n-1}}|a(y)|\left|\frac{1}{|x-y|^{2}}-\frac{1}{(|x|+2 r)^{2}}\right|^{1 / 2} d y\right)^{p} d x \\
& \leq C r^{\frac{p}{2}} \int_{\left(B^{*}\right)^{c}}\left(\int_{B} \frac{|\Omega(x, x-y)|}{|x-y|^{n+\frac{1}{2}}}|a(y)| d y\right)^{p} d x .
\end{aligned}
$$

Since $1>p>\frac{n}{n+\frac{1}{2}}$, so we can choose $\varepsilon$ satisfying $0<\varepsilon<n+\frac{1}{2}-\frac{n}{p}$. Using Hölder inequality for integrals, we have

$$
I_{1} \leq C r^{\frac{p}{2}} \int_{\left(B^{*}\right)^{c}}\left(\int_{B} \frac{|\Omega(x, x-y)|}{|x-y|^{n+\varepsilon}} \cdot|x-y|^{\varepsilon-\frac{1}{2}}|a(y)| d y\right)^{p} d x
$$




$$
\begin{aligned}
& =C r^{\frac{p}{2}} \int_{\left(B^{*}\right)^{c}}\left(\int_{B} \frac{|\Omega(x, x-y)|}{|x-y|^{n+\varepsilon}} \cdot|a(y)| d y\right)^{p} \cdot|x|^{\left(\varepsilon-\frac{1}{2}\right) p} d x \\
& \leq C r^{\frac{p}{2}}\left(\int_{\left(B^{*}\right)^{c}} \int_{B} \frac{|\Omega(x, x-y)|}{|x-y|^{n+\varepsilon}} \cdot|a(y)| d y d x\right)^{p}\left(\int_{\left(B^{*}\right)^{c}}|x|^{\frac{\left(\varepsilon-\frac{1}{2}\right) p}{1-p}} d x\right)^{1-p} \\
& \leq C r^{\frac{p}{2}}\left(\int_{B} \int_{\left(B^{*}\right)^{c}} \frac{|\Omega(x, x-y)|}{|x-y|^{n+\varepsilon}} d x \cdot|a(y)| d y\right)^{p}\left(\int_{8 r}^{\infty} t^{\frac{\left(\varepsilon-\frac{1}{2}\right)_{p}}{1-p}} \cdot t^{n-1} d t\right)^{1-p} \\
& \leq C r^{\frac{p}{2}}\|\Omega\|_{L^{\infty} \times L^{1}}^{p}\left(\int_{B}|a(y)| d y \int_{8 r}^{+\infty} t^{-n-\varepsilon} t^{n-1} d t\right)^{p} \cdot r^{\left(\varepsilon-\frac{1}{2}\right) p+n(1-p)} \\
& \leq C r^{n(1-p)}\left(\int_{B}|a(y)| d y\right)^{p} \leq C r^{n(1-p)}|B|^{\frac{p}{2}}\|a\|_{L^{2}}^{p} \leq C .
\end{aligned}
$$

where we have used that $|x-y| \sim|x|$.

As to the estimate of $I_{2}$. Noting that if $t \geq|x|+2 r$, then $B \subset\{y$ : $|x-y|<t\}$. So by the cancellation condition (iii) of $a$, we have

$$
\int_{|x-y|<t} a(y) d y=0 .
$$

From this and Minkowski's inequality for integrals, we obtain

$$
\begin{aligned}
& I_{2}=\int_{\left(B^{*}\right)^{c}}\left(\int_{|x|+2 r}^{\infty} \mid \int_{|x-y| \leq t}\left(\frac{\Omega(x, x-y)}{|x-y|^{n-1}}-\frac{\Omega(x, x)}{|x-y|^{n-1}}\right.\right. \\
& \left.\left.\quad+\frac{\Omega(x, x)}{|x-y|^{n-1}}-\frac{\Omega(x, x)}{|x|^{n-1}}\right)\left.\cdot a(y) d y\right|^{2} \frac{1}{t^{3}} d t\right)^{p / 2} d x \\
& \leq I_{21}+I_{22},
\end{aligned}
$$

where

$I_{21}=\int_{\left(B^{*}\right)^{c}}\left(\int_{|x|+2 r}^{\infty}\left|\int_{|x-y| \leq t} \frac{|\Omega(x, x-y)-\Omega(x, x)|}{|x-y|^{n-1}}\right| a(y)|d y|^{2} \frac{1}{t^{3}} d t\right)^{p / 2} d x$

and

$I_{22}=\int_{\left(B^{*}\right)^{c}}\left(\int_{|x|+2 r}^{\infty}\left|\int_{|x-y| \leq t}\right| \frac{\Omega(x, x)}{|x-y|^{n-1}}-\frac{\Omega(x, x)}{|x|^{n-1}}|| a(y)|d y|^{2} \frac{1}{t^{3}} d t\right)^{p / 2} d x$. 
Applying Minkowski inequality for integrals, Hölder inequality for integrals and Fubini theorem successively, we can obtain

$$
\begin{aligned}
& I_{21} \leq \int_{\left(B^{*}\right)^{c}}\left|\int_{B} \frac{|\Omega(x, x-y)-\Omega(x, x)|}{|x-y|^{n}}\right| a(y)|d y|^{p} d x \\
& \leq \sum_{j=3}^{+\infty} \int_{2^{j} r \leq|x|<2^{j+1_{r}}}\left|\int_{B} \frac{|\Omega(x, x-y)-\Omega(x, x)|}{|x-y|^{n}}\right| a(y)|d y|^{p} d x \\
& \leq \sum_{j=3}^{+\infty}\left(\int_{2^{j} r \leq|x|<2^{j+1} r} \int_{B} \frac{|\Omega(x, x-y)-\Omega(x, x)|}{|x-y|^{n}}|a(y)| d y d x\right)^{p}\left(2^{j} r\right)^{n(1-p)} \\
& =\sum_{j=3}^{+\infty}\left(\int_{B}\left(\int_{2^{j} r \leq|x|<2^{j+1} r} \frac{|\Omega(x, x-y)-\Omega(x, x)|}{|x-y|^{n}} d x\right)|a(y)| d y\right)^{p}\left(2^{j} r\right)^{n(1-p)} .
\end{aligned}
$$

To estimate the inner integral above, we note $|y|<r$ and $|x|>2 r$, which implies,

$$
\begin{gathered}
\left|\frac{x-y}{|x-y|}-\frac{x}{|x|}\right|=\left|\frac{(|x|-|x-y|) x-|x| y}{|x||x-y|}\right| \\
\leq \frac{|| x|-| x-y||+|y|}{|x-y|} \leq 4 \frac{|y|}{|x|} .
\end{gathered}
$$

And thus

$$
\begin{aligned}
& \int_{\mathbb{S}^{n-1}}|\Omega(x, x-y)-\Omega(x, x)| d \sigma\left(x^{\prime}\right) \\
& =\int_{\mathbb{S}^{n-1}}\left|\Omega\left(x, \frac{x-y}{|x-y|}\right)-\Omega\left(x, \frac{x}{|x|}\right)\right| d \sigma\left(x^{\prime}\right) \leq \varpi\left(4 \frac{|y|}{|x|}\right) .
\end{aligned}
$$

This and a direct computation give

$$
\begin{aligned}
& \int_{2^{j} r \leq|x|<2^{j+1} r} \frac{|\Omega(x, x-y)-\Omega(x, x)|}{|x-y|^{n}} d x \\
& \leq C \int_{2^{j} r}^{2^{j+1} r} t^{-1} \varpi\left(\frac{4|y|}{t}\right) d t \\
& =C \int_{\frac{|y|}{2^{j}-1_{r}}}^{\frac{|y|}{2^{j-2} r}} \frac{\varpi(\delta)}{\delta} d \delta .
\end{aligned}
$$

Now using the condition $\int_{0}^{1} \frac{\varpi(\delta)}{\delta^{1+\alpha}} d \delta<\infty$, and $1>p>\frac{n}{n+\alpha}$, we can get 


$$
\begin{aligned}
I_{21} & \leq C \sum_{j=3}^{+\infty}\left(\int_{B}|a(y)| \int_{\frac{|y|}{2^{j-1} r}}^{\frac{|y|}{2^{-2} r}} \frac{\varpi(\delta)}{\delta^{1+\alpha}} \delta^{\alpha} d \delta d y\right)^{p}\left(2^{j} r\right)^{n(1-p)} \\
& \leq C \sum_{j=3}^{+\infty}\left(2^{j} r\right)^{n(1-p)}\left(2^{j}\right)^{-p \alpha}\left(\int_{B}|a(y)| d y\right)^{p} \\
& \leq C r^{n(1-p)}\left(\int_{B}|a(y)| d y\right)^{p} \leq C .
\end{aligned}
$$

At last, we give the estimate of $I_{22}$. Obviously, mean value theorem gives

$$
\left|\frac{1}{|x-y|^{n-1}}-\frac{1}{|x|^{n-1}}\right| \leq C \frac{|y|}{|x|^{n}}
$$

Applying this inequality, Minkowski's inequality for integrals, and the fact $\frac{n}{n+1}<p<1$, we deduce that

$$
\begin{aligned}
I_{22} & \leq \int_{|x|>8 r}\left(\int_{|x|+2 r}^{\infty}\left(\int_{|x-y| \leq t} \frac{|\Omega(x, x)||y|}{|x|^{n}}|a(y)| d y\right)^{2} \frac{d t}{t^{3}}\right)^{p / 2} d x \\
& \leq C \int_{|x|>8 r}\left(\int_{B} \frac{|\Omega(x, x)||y|}{|x|^{n+1}}|a(y)| d y\right)^{p} d x \\
& \leq C r^{p}\left(\int_{|x|>8 r}|\Omega(x, x)|^{p}|x|^{(-n-1) p} d x\right)\left(\int_{B}|a(y)| d y\right)^{p} \\
& \leq C r^{p} r^{(-n-1) p+n}\|\Omega\|_{L^{\infty} \times L^{p}}^{p}\left(\int_{B}|a(y)| d y\right)^{p} \\
& \leq C .
\end{aligned}
$$

Combining (2.3), and the estimates of $I_{1}, I_{21}$ and $I_{22}$, we get (2.2) and then complete the proof of Theorem 1.1.

\section{Proof of theorem 1.4}

In order to prove theorem 1.4, we need the following decomposition theorem for distributions in $H^{p, \infty}\left(\mathbb{R}^{n}\right)$.

Lemma 3.1. [10] Given a distribution $f \in H^{p, \infty}\left(\mathbb{R}^{n}\right)$, there exits a bounded function sequence $\left\{f_{k}\right\}_{k=-\infty}^{+\infty}$ which has the following properties:

(a) $f=\sum_{k} f_{k}$ in the sense of distributions; 
(b) Each $f_{k}$ may be further decomposed in $L^{p}$ as $f_{k}=\sum_{i} h_{i}^{k}$, where $\left\{h_{i}^{k}\right\}$ satisfies the following three conditions:

(i) $\operatorname{supp}\left(h_{i}^{k}\right) \subset B_{i}^{k}:=B\left(x_{i}^{k}, r_{i}^{k}\right)$, where $B(x, r)$ denotes the ball in $\mathbb{R}^{n}$ with the center at $x$ and radius $r$. Moreover, $\sum_{i}\left|B_{i}^{k}\right| \leq C_{1} 2^{-k p}$, where $C_{1} \sim\|f\|_{H^{p, \infty}}^{p}$ and $\sum_{i} \chi_{B_{i}^{k}}(x) \leq C$;

(ii) $\left\|h_{i}^{k}\right\|_{\infty} \leq C 2^{k}$;

(iii) $\int h_{i}^{k}(x) d x=0$.

We now give the proof of Theorem 1.4. For any $f \in H^{p, \infty}\left(\mathbb{R}^{n}\right)$ and $\beta>0$, we choose $k_{0}$ satisfying $2^{k_{0}} \leq \beta<2^{k_{0}+1}$. Applying Lemma 3.1, we can write

$$
f=\sum_{k=-\infty}^{k_{0}} f_{k}+\sum_{k=k_{0}+1}^{+\infty} f_{k}=F_{1}+F_{2}, \quad f_{k}=\sum_{i} h_{i}^{k}
$$

where $h_{i}^{k}$ satisfies the properties (i), (ii) and (iii) of Lemma 3.1.

Let $A_{k}=\operatorname{supp}\left(f_{k}\right)$, then $A_{k}=\bigcup_{i} B_{i}^{k}$ and $\left|A_{k}\right| \leq \sum_{i}\left|B_{i}^{k}\right| \leq$ $C 2^{-k p}\|f\|_{H^{p, \infty}}^{p}$, also $\left\|f_{k}\right\|_{\infty} \leq C 2^{k}$, so we can get

$$
\begin{aligned}
\left\|F_{1}\right\|_{2} \leq & \sum_{k=-\infty}^{k_{0}}\left\|f_{k}\right\|_{2} \leq C \sum_{k=-\infty}^{k_{0}} 2^{k}\left|A_{k}\right|^{\frac{1}{2}} \\
& \leq \sum_{k=-\infty}^{k_{0}} 2^{k\left(1-\frac{p}{2}\right)}\|f\|_{H^{p, \infty}}^{\frac{p}{2}} \leq C\|f\|_{H^{p, \infty}}^{\frac{p}{2}} \beta^{1-\frac{p}{2}}
\end{aligned}
$$

By the $L^{2}$-boundedness of $\mu_{\Omega}$, it follows

$$
\left|\left\{x: \mu_{\Omega}\left(F_{1}\right)(x)>\beta\right\}\right| \leq \frac{\left\|\mu_{\Omega}\left(F_{1}\right)\right\|_{2}^{2}}{\beta^{2}} \leq C \frac{\left\|F_{1}\right\|_{2}^{2}}{\beta^{2}} \leq C \frac{\|f\|_{H^{p, \infty}}^{p}}{\beta^{p}} .
$$

On the other hand, we denote $\bar{B}_{i}^{k}=B\left(x_{i}^{k}, 2 R_{i}^{k}\right)$ and $R_{i}^{k}=\left(\frac{4}{3}\right)^{\frac{\left(k-k_{0}\right)}{n}} r_{i}^{k}$, and let $B_{k_{0}}=\bigcup_{k=k_{0}+1}^{+\infty} \bigcup_{i} \bar{B}_{i}^{k}$, we can get

$$
\left|B_{k_{0}}\right| \leq C \sum_{k=k_{0}+1} \sum_{i}\left|\bar{B}_{i}^{k}\right| \leq C \sum_{k=k_{0}+1} \sum_{i} 2^{n}\left(\frac{4}{3}\right)^{k-k_{0}}\left|B_{i}^{k}\right|
$$

$$
\leq C \sum_{k=k_{0}+1}^{+\infty} \sum_{i}\left(\frac{4}{3}\right)^{k-k_{0}}\left|B_{i}^{k}\right| \leq C \sum_{k=k_{0}+1}^{+\infty}\left(\frac{4}{3}\right)^{k-k_{0}} 2^{-k p}\|f\|_{H^{p, \infty}}^{p} \leq C \frac{\|f\|_{H^{p, \infty}}^{p}}{\beta^{p}},
$$


where the last inequality holds owing to $p>\frac{n}{n+\frac{1}{2}}$. Therefore, in order to prove Theorem 1.4, it suffices to show

$$
\left|\left\{x \in\left(B_{k_{0}}\right)^{c}: \mu_{\Omega}\left(F_{2}\right)(x)>\beta\right\}\right| \leq C \frac{\|f\|_{H^{p, \infty}}^{p}}{\beta^{p}} .
$$

Firstly, a similar argument as the one used in (2.1) and Minkowski's inequality for series give

$$
\begin{aligned}
\int_{\left(B_{k_{0}}\right)^{c}}\left|\mu_{\Omega}\left(F_{2}\right)(x)\right|^{p} d x & \leq \int_{\left(B_{k_{0}}\right)^{c}} \sum_{k=k_{0}+1}^{+\infty} \sum_{i}\left|\mu_{\Omega}\left(h_{i}^{k}\right)(x)\right|^{p} d x \\
& \leq C \sum_{k=k_{0}+1}^{+\infty} \sum_{i}\left(J_{1}+J_{2}\right)
\end{aligned}
$$

where

$$
J_{1}=\left.\left.\int_{\left(B_{k_{0}}\right)^{c}}\left|\int_{0}^{\left|x-x_{i}^{k}\right|+2 r_{i}^{k}}\right| \int_{|x-y| \leq t} \frac{\Omega(x, x-y)}{|x-y|^{n-1}} h_{i}^{k}(y) d y\right|^{2} \frac{d t}{t^{3}}\right|^{\frac{p}{2}} d x,
$$

and

$$
J_{2}=\left.\left.\int_{\left(B_{k_{0}}\right)^{c}}\left|\int_{\left|x-x_{i}^{k}\right|+2 r_{i}^{k}}^{+\infty}\right| \int_{|x-y| \leq t} \frac{\Omega(x, x-y)}{|x-y|^{n-1}} h_{i}^{k}(y) d y\right|^{2} \frac{d t}{t^{3}}\right|^{\frac{p}{2}} d x .
$$

It is obvious that when $y \in B_{i}^{k}, x \in B_{k_{0}}^{c}$, we will have $\left|x-x_{i}^{k}\right| \sim|x-y| \sim$ $\left|x-x_{i}^{k}\right|+2 r_{i}^{k}$ and

$$
\left|\frac{1}{\left(\left|x-x_{i}^{k}\right|+2 r_{i}^{k}\right)^{2}}-\frac{1}{|x-y|^{2}}\right| \leq C \frac{r_{i}^{k}}{|x-y|^{3}} .
$$

Because of $\frac{n}{n+\frac{1}{2}}<p<1$, one can choose proper $\varepsilon$ such that $0<\varepsilon<$ $n+\frac{1}{2}-\frac{n}{p}$. Combining the above inequality and Minkowski's inequality for integrals, the size condition of $h_{i}^{k}$ and Hölder inequality for integrals, we deduce that

$$
\begin{aligned}
J_{1} & \leq \int_{\left(B_{k_{0}}\right)^{c}}\left|\int_{B_{i}^{k}} \frac{|\Omega(x, x-y)|}{|x-y|^{n-1}}\right| h_{i}^{k}(y)\left|\frac{\left(r_{i}^{k}\right)^{\frac{1}{2}}}{|x-y|^{\frac{3}{2}}} d y\right|^{p} d x \\
& \leq C 2^{k p}\left(r_{i}^{k}\right)^{\frac{p}{2}} \int_{\left(B_{k_{0}}\right)^{c}}\left|\int_{B_{i}^{k}} \frac{|\Omega(x, x-y)|}{|x-y|^{n+\varepsilon}}\right| x-\left.\left.x_{i}^{k}\right|^{\varepsilon-\frac{1}{2}} d y\right|^{p} d x
\end{aligned}
$$




$$
\begin{aligned}
& =C 2^{k p}\left(r_{i}^{k}\right)^{\frac{p}{2}} \int_{\left(B_{k_{0}}\right)^{c}}\left|\int_{B_{i}^{k}} \frac{|\Omega(x, x-y)|}{|x-y|^{n+\varepsilon}} d y\right|^{p}\left|x-x_{i}^{k}\right|^{\left(\varepsilon-\frac{1}{2}\right) p} d x \\
& \leq C 2^{k p}\left(r_{i}^{k}\right)^{\frac{p}{2}}\left(\int_{\left(B_{k_{0}}\right)^{c}} \int_{B_{i}^{k}} \frac{|\Omega(x, x-y)|}{|x-y|^{n+\varepsilon}} d y d x\right)^{p}\left(\int_{\left(B_{k_{0}}\right)^{c}}\left|x-x_{i}^{k}\right|^{\frac{p\left(\varepsilon-\frac{1}{2}\right)}{1-p}} d x\right)^{1-p} \\
& =C 2^{k p}\left(r_{i}^{k}\right)^{\frac{p}{2}}\left(\int_{B_{i}^{k}}\left|\int_{\left(B_{k_{0}}\right)^{c}} \frac{|\Omega(x, x-y)|}{|x-y|^{n+\varepsilon}} d x\right| d y\right)^{p}\left(\int_{2 R_{i}^{k}}^{+\infty} r^{\frac{\left(\varepsilon-\frac{1}{2}\right) p}{1-p}+n-1} d r\right)^{1-p} \\
& \leq C 2^{k p}\left(r_{i}^{k}\right)^{\frac{p}{2}}\left|B_{i}^{k}\right|^{p}\|\Omega\|_{L^{\infty} \times L^{1}}^{p}\left(\int_{2 R_{i}^{k}}^{+\infty} r^{-\varepsilon-1} d r\right)^{p}\left(2 R_{i}^{k}\right)^{\left(\varepsilon-\frac{1}{2}\right) p+n(1-p)} \\
& \leq C 2^{k p}\left(r_{i}^{k}\right)^{\frac{p}{2}}\left|B_{i}^{k}\right|^{p}\left(2\left(\frac{4}{3}\right)^{\frac{k-k_{0}}{n}} r_{i}^{k}\right)^{n(1-p)-\frac{p}{2}} \leq C 2^{k p}\left|B_{i}^{k}\right|\left(\frac{3}{4}\right)^{\frac{\left(k-k_{0}\right)\left(n p+\frac{p}{2}-n\right)}{n}} .
\end{aligned}
$$

It follows

$$
\sum_{k=k_{0}+1}^{+\infty} \sum_{i} J_{1} \leq C \sum_{k=k_{0}+1}^{+\infty}\left(\frac{3}{4}\right)^{\frac{\left(k-k_{0}\right)\left(n p+\frac{p}{2}-n\right)}{n}}\|f\|_{H^{p, \infty}}^{p} \leq C\|f\|_{H^{p, \infty}}^{p}
$$

Next we estimate $I_{2}$. One notes that $B_{i}^{k} \subset\{y ;|x-y|<t\}$ since $x \in B_{k_{0}}^{c}$ and $t>\left|x-x_{i}^{k}\right|+2 r_{i}^{k}$. By the cancellation of $h_{i}^{k}$ and Minkowski's inequality for integrals, we can get

$$
\begin{aligned}
J_{2}= & \left.\left.\int_{\left(B_{k_{0}}\right)^{c}}\left|\int_{\left|x-x_{i}^{k}\right|+2 r_{i}^{k}}^{+\infty}\right| \int_{|x-y| \leq t} \frac{\Omega(x, x-y)}{|x-y|^{n-1}} h_{i}^{k}(y) d y\right|^{2} \frac{d t}{t^{3}}\right|^{\frac{p}{2}} d x \\
= & \int_{\left(B_{k_{0}}\right)^{c}}\left|\int_{\left|x-x_{i}^{k}\right|+2 r_{i}^{k}}^{+\infty}\right| \int_{|x-y| \leq t}\left(\frac{\Omega(x, x-y)}{|x-y|^{n-1}}-\frac{\Omega(x, x-y)}{\left|x-x_{i}^{k}\right|^{n-1}}\right. \\
& \left.\quad+\frac{\Omega(x, x-y)}{\left|x-x_{i}^{k}\right|^{n-1}}-\frac{\Omega\left(x, x-x_{i}^{k}\right)}{\left|x-x_{i}^{k}\right|^{n-1}}\right)\left.\left.h_{i}^{k}(y) d y\right|^{2} \frac{d t}{t^{3}}\right|^{\frac{p}{2}} d x \\
\leq & C 2^{k p} \int_{\left(B_{k_{0}}\right)^{c}}\left|\frac{1}{\left|x-x_{i}^{k}\right|} \int_{B_{i}^{k}}\right| \frac{\Omega(x, x-y)}{\left|x-x_{i}^{k}\right|^{n-1}}-\frac{\Omega\left(x, x-x_{i}^{k}\right)}{\left|x-x_{i}^{k}\right|^{n-1}}|d y|^{p} d x \\
& +C 2^{k p} \int_{\left(B_{k_{0}}\right)^{c}}\left|\frac{1}{\left|x-x_{i}^{k}\right|} \int_{B_{i}^{k}}\right| \frac{\Omega(x, x-y)}{|x-y|^{n-1}}-\frac{\Omega(x, x-y)}{\left|x-x_{i}^{k}\right|^{n-1}}|d y|^{p} d x \\
= & C 2^{k p}\left(J_{2,1}+J_{2,2}\right) .
\end{aligned}
$$


Decompose $J_{2,1}$ as following

$$
\begin{array}{r}
J_{2,1}=\sum_{j=1}^{+\infty} \int_{2^{j} R_{i}^{k} \leq\left|x-x_{i}^{k}\right| \leq 2^{j+1} R_{i}^{k}}\left|\int_{B_{i}^{k}}\right| \frac{\Omega(x, x-y)}{\left|x-x_{i}^{k}\right|^{n}}-\frac{\Omega\left(x, x-x_{i}^{k}\right)}{\left|x-x_{i}^{k}\right|^{n}}|d y|^{p} d x \\
\leq \sum_{j=1}^{+\infty}\left(2^{j} R_{i}^{k}\right)^{n(1-p)}\left(\int_{2^{j} R_{i}^{k} \leq\left|x-x_{i}^{k}\right| \leq 2^{j+1} R_{i}^{k}} \mid \int_{B_{i}^{k}} \frac{\Omega(x, x-y)}{\left|x-x_{i}^{k}\right|^{n}}\right. \\
\left.-\frac{\Omega\left(x, x-x_{i}^{k}\right)}{\left|x-x_{i}^{k}\right|^{n}}|d y| d x\right)^{p} .
\end{array}
$$

Observe that $\left|y-x_{i}^{k}\right|<r_{i}^{k}$ and $\left|x-x_{i}^{k}\right|>R_{i}^{k} \geq 2 r_{i}^{k}$. Using a similar argument as in inequalities (2.4) and (2.5), we can deduce that

$$
\left|\frac{x-y}{|x-y|}-\frac{x-x_{i}^{k}}{\left|x-x_{i}^{k}\right|}\right| \leq 4 \frac{\left|y-x_{i}^{k}\right|}{\left|x-x_{i}^{k}\right|}
$$

and then

$$
\begin{aligned}
& \int_{2^{j} R_{i}^{k} \leq\left|x-x_{i}^{k}\right| \leq 2^{j+1} R_{i}^{k}}\left|\frac{\Omega(x, x-y)}{\left|x-x_{i}^{k}\right|^{n}}-\frac{\Omega\left(x, x-x_{i}^{k}\right)}{\left|x-x_{i}^{k}\right|^{n}}\right| d x \\
& =\int_{2^{j} R_{i}^{k}}^{2^{j+1} R_{i}^{k}} \int_{\mathbb{S}^{n-1}}\left|\Omega\left(r x^{\prime}+x_{i}^{k}, \frac{r x^{\prime}+x_{i}^{k}-y}{\left|r x^{\prime}+x_{i}^{k}-y\right|}\right)-\Omega\left(r x^{\prime}+x_{i}^{k}, r x^{\prime}\right)\right| d \sigma\left(x^{\prime}\right) r^{-1} d r \\
& \leq C \int_{2^{j} R_{i}^{k}}^{2^{j+1} R_{i}^{k}} \varpi\left(\frac{4\left|y-x_{i}^{k}\right|}{r}\right) r^{-1} d r \\
& =C \int_{\frac{\left|y-x_{i}^{k}\right|}{2^{j-1} R_{i}^{k}}}^{\frac{\left|y-x_{i}^{k}\right|}{2^{j-2} R_{k}^{k}}}\left(\frac{\left|y-x_{i}^{k}\right|}{\delta}\right)^{-1} \varpi(\delta) \frac{\left|y-x_{i}^{k}\right|}{\delta^{2}} d \delta \\
& =C \int_{\frac{\left|y-x_{i}^{k}\right|}{2^{j-1} R_{i}^{k}}}^{\frac{\left|y-x_{i}^{k}\right|}{2^{j-2} R_{i}^{k}}} \frac{\varpi(\delta)}{\delta^{1+\alpha}} \delta^{\alpha} d \delta \leq C\left(\frac{r_{i}^{k}}{2^{j} R_{i}^{k}}\right)^{\alpha} .
\end{aligned}
$$

This follows

$$
\begin{aligned}
J_{2,1} & \leq C \sum_{j=1}^{+\infty}\left(2^{j} R_{i}^{k}\right)^{n(1-p)}\left(\frac{r_{i}^{k}}{2^{j} R_{i}^{k}}\right)^{\alpha p}\left|B_{i}^{k}\right|^{p} \\
& \leq C\left|B_{i}^{k}\right|\left(\frac{3}{4}\right)^{\frac{k-k_{0}}{n}(n p-n+\alpha p)} \cdot \sum_{j=1}^{+\infty} 2^{j(n-n p-\alpha p)} \leq C\left|B_{i}^{k}\right|\left(\frac{3}{4}\right)^{\frac{k-k_{0}}{n}(n p-n+\alpha p)} .
\end{aligned}
$$


To deal with $J_{2,2}$, we use the mean value theorem, Hölder inequality for integrals, and the condition $\frac{n}{n+\frac{1}{2}}<p<1$ to give

$$
\begin{aligned}
J_{2,2} & =\int_{\left(B_{k_{0}}\right)^{c}}\left(\frac{1}{\left|x-x_{i}^{k}\right|} \int_{B_{i}^{k}}\left|\frac{\Omega(x, x-y)}{|x-y|^{n-1}}-\frac{\Omega(x, x-y)}{\left|x-x_{i}^{k}\right|^{n-1}}\right| d y\right)^{p} d x \\
& \leq C \int_{\left(B_{k_{0}}\right)^{c}}\left(\frac{1}{\left|x-x_{i}^{k}\right|} \int_{B_{i}^{k}} \frac{|\Omega(x, x-y)|}{\left|x-x_{i}^{k}\right|^{n}} r_{i}^{k} d y\right)^{p} d x \\
& =C\left(r_{i}^{k}\right)^{p} \int_{\left(B_{k_{0}}\right)^{c}}\left|x-x_{i}^{k}\right|^{-\frac{p}{2}}\left(\int_{B_{i}^{k}} \frac{|\Omega(x, x-y)|}{\left|x-x_{i}^{k}\right|^{n+\frac{1}{2}}} d y\right)^{p} d x \\
& \leq\left(r_{i}^{k}\right)^{p}\left(\int_{\left(B_{k_{0}}\right)^{c}}\left|\int_{B_{i}^{k}} \frac{|\Omega(x, x-y)|}{\left|x-x_{i}^{k}\right|^{n+\frac{1}{2}}} d y\right| d x\right)^{p}\left(\int_{\left(B_{k_{0}}\right)^{c}}\left|x-x_{i}^{k}\right|^{-\frac{p}{2(1-p)}} d x\right)^{1-p}=C\left|B_{i}^{k}\right|\left(\frac{3}{4}\right)^{\frac{\left(k-k_{0}\right)(n p+p-n)}{n}} . \\
& \leq C\left(r_{i}^{k}\right)^{p}\left|B_{i}^{k}\right|^{p}\left(2\left(\frac{4}{3}\right)^{\frac{k-k_{0}}{n}} r_{i}^{k}\right)^{-\frac{p}{2}+n(1-p)} .
\end{aligned}
$$

Combining the estimates of $J_{2,1}$ and $J_{2,1}$, we obtain that

$$
\begin{aligned}
& \sum_{k=k_{0}+1}^{+\infty} \sum_{i} J_{2} \leq C \sum_{k=k_{0}+1}^{+\infty} \sum_{i} 2^{k p}\left(J_{2,1}+J_{2,2}\right) \\
& \leq C \sum_{k=k_{0}+1}^{+\infty}\left(\left(\frac{3}{4}\right)^{\frac{\left(k-k_{0}\right)(n p+\alpha p-n)}{n}}+\left(\frac{3}{4}\right)^{\frac{\left(k-k_{0}\right)(n p+p-n)}{n}}\right)\|f\|_{H^{p, \infty}}^{p} \\
& \leq C\|f\|_{H^{p, \infty}}^{p} .
\end{aligned}
$$

Obviously, the inequalities (3.5), (3.6) and (3.4) yield the inequality (3.3) and the result follows.

\section{References}

[1] A. Al-Salman, H. Al-Qassem, L. C. Cheng and Y. Pan, $L^{p}$ bounds for the function of Marcinkiewicz, Math. Research Letter, 9 (2002), $697-700$.

[2] N.E. Aguilera and E.O. Harboure, Some inequalities for maximal operators, Indiana. Univ. Math. J., 29 (1980), 559-576.

[3] A. Benedek, A.P. Calderon and R. Panzone, Convolution operators on Banach space valued functions, Proc. Nat. Acad. Sci. U.S.A., 48 (1962), 356-365. 
[4] A.P. Calderón and A. Zygmund, On a problem of Mihlin, Trans. Amer. Math. Soc., 78 (1955), 209-224.

[5] A.P. Calderón and A. Zygmund, On singular integral with variable kernels, Applicable Anal., 7 (1977/1978), 221-238.

[6] J.-C. Chen, D.-S. Fan and Y.-M. Ying, Certain operators with rough singular kernels, Canad. J. Math., 55 (2003), 504-532.

[7] Y. Ding, C.-C. Lin, and S.L. Shao, On Marcinkiewicz integral with variable kernels, Indiana. Univ. Math. J., 53 (2004), 805-821.

[8] Y. Ding, S. Lu, and Q. Xue, Marcinkiewicz integral on Hardy spaces, Integral Equations and Operator Theory, 42 (2002), 174-182.

[9] J. Garcia-Cuerva and J. Rubio de Francia, Weighted Norm Inequalities and Related Topics, North Holland, 1985.

[10] H.-P. Liu, The weak $H^{p}$ space on homogeneous groups, Lecture notes in Math, 1494 (1991), 113-118.

[11] M. Sakamoto and K. Yabuta, Boundedness of Marcinkiewicz functions, Studia Math., 135 (1999), 103-142.

[12] E.M. Stein, On the function of Littlewood-Paley, Lusin and Marcinkiewicz, Trans. Amer. Math. Soc., 88 (1958), 430-466.

[13] E.M. Stein, Harmonic Analysis: Real Variable Methods, Orthogonality and Oscillatory Integrals, Princeton Univ. Press, (1993).

[14] X.-X. Tao and Q. Chen, The boundedness of maximal function in Orlicz-Campanato spaces of homogeneous type, Georgian Math. J.,15 (2008),377-388.

[15] X.-X. Tao, X. Yu and S.-Y. Zhang, Boundedness on Hardy-Sobolev Spaces for Hypersingular Marcinkiewicz Integrals with Variable Kernels, J. Inequal. Appl., (2008), Article ID 835938, 17 pages.

[16] X.-X. Tao, R.-Y. Wei and S.-Y. Zhang, $H^{p}$ bounds for some Littlewood-Paley operators with rough variable kernels, African Diaspora J. Math., 8 (2009),16-27.

Department of Mathematics

Zhejiang University of Science\& Technology

Hangzhou, Zhejiang province, 310023

P. R. China

(E-mail : xxtau@163.com)

(E-mail : yx2000s@sinra.com)

(E-mail : syzh201@163.com)

(Received: July 2007) 


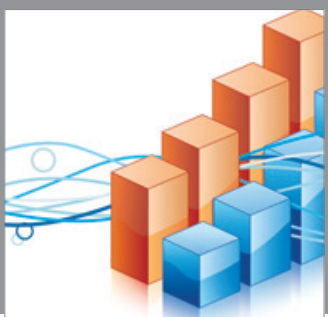

Advances in

Operations Research

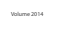

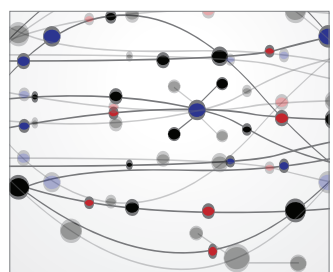

\section{The Scientific} World Journal
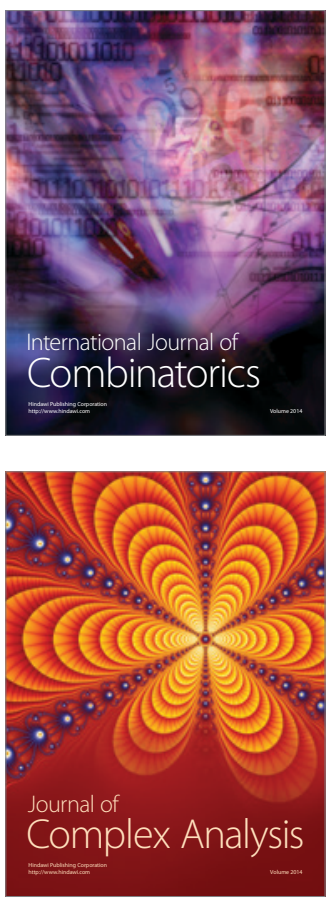

International Journal of

Mathematics and

Mathematical

Sciences
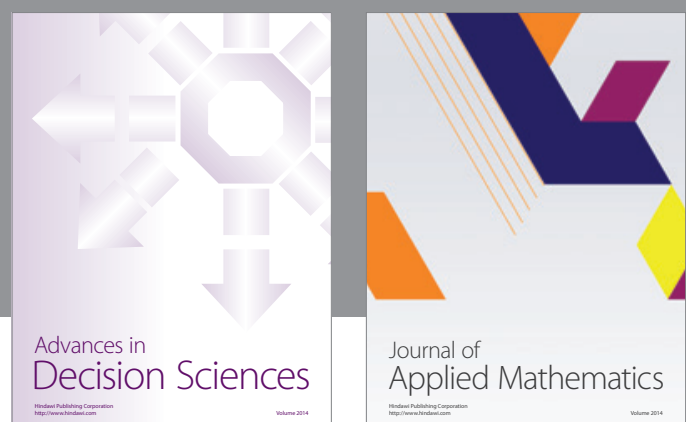

Journal of

Applied Mathematics
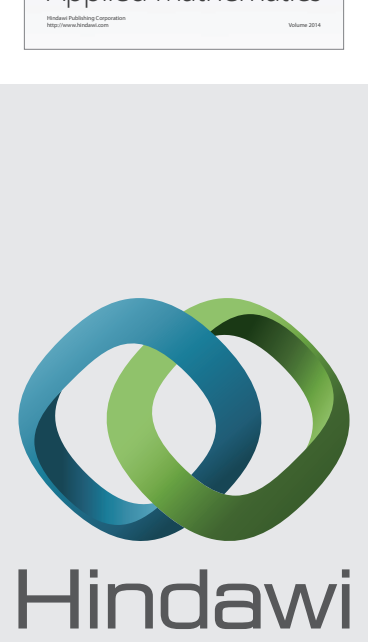

Submit your manuscripts at http://www.hindawi.com
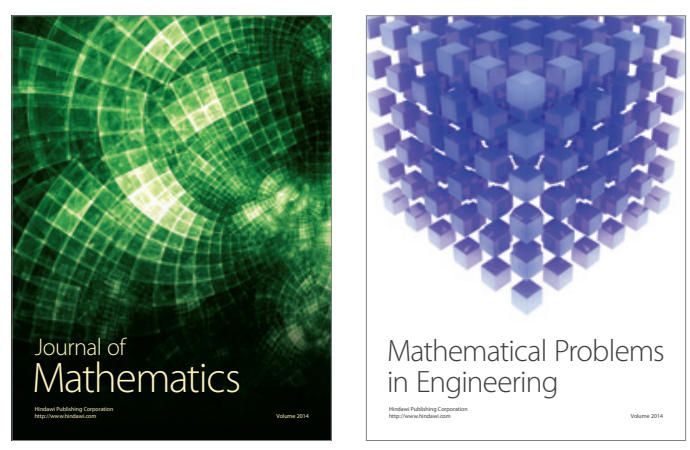

Mathematical Problems in Engineering
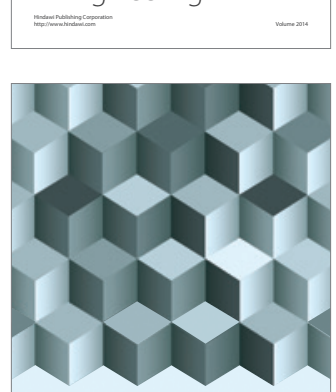

Journal of

Function Spaces
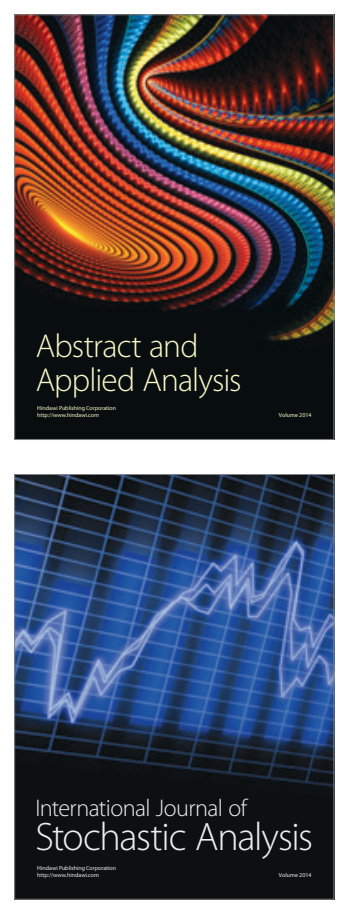

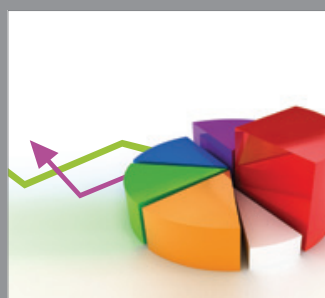

ournal of

Probability and Statistics

Promensencen
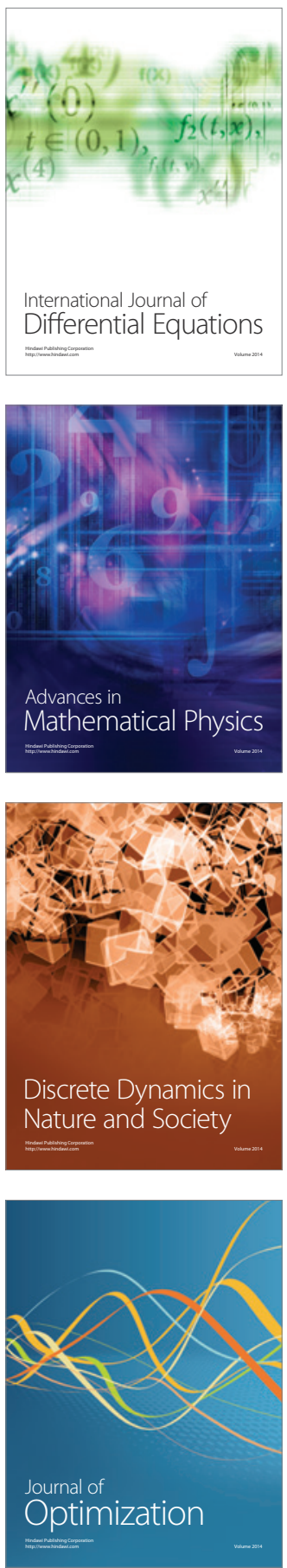